\title{
[editorial]
}

\section{Compromiso oral en Esclerosis Sistémica}

\author{
Marina Scolnik
}

Médica de planta, Sección Reumatología, Hospital Italiano de Buenos Aires.

El tracto gastrointestinal (GI) está afectado en hasta el 90\% de los pacientes con Esclerosis Sistémica $(\mathrm{ES})^{1-3}$. Los síntomas son variados de acuerdo al sitio y grado de compromiso. Disfagia, reflujo, náuseas, vómitos, dolor abdominal, diarrea, constipación, incontinencia fecal y pérdida de peso son los síntomas más comúnmente reportados. El compromiso GI afecta severamente la calidad de vida y es una de las mayores causas de morbimortalidad de la enfermedad ${ }^{4}$.

Mientras que el esófago es el sitio mayoritariamente comprometido, es sabido que la ES puede afectar cualquier sitio del tracto GI desde la boca hasta el ano.

El compromiso GI ha sido observado con una frecuencia similar en las formas limitadas y difusas de la enfermedad en varias cohortes de pacientes ${ }^{5}$.

Las manifestaciones orofaríngeas en ES no han sido bien estudiadas y se estima que suceden en un 20 a $80 \%$ de los pacientes ${ }^{6-8}$. La esclerosis de la mucosa orofaríngea, de los músculos asociados con la masticación y de las glándulas salivales puede conducir a dificultades en el habla, la masticación y la deglución. Los problemas reportados incluyen fibrosis de la lengua y el paladar, microstomía, daño de la mucosa oral, daño cutáneo perioral, xerostomía, engrosamiento fibroso ligamentario periodontal, reabsorción ósea, telangiectasias orales, neuropatía del trigémino y aumento significativo de caries ${ }^{9}$. El compromiso cutáneo importante a nivel perioral limita la apertura bucal y puede restringir la ingesta.

Los síntomas sicca se encuentran en un número variable de pacientes, los reportes hablan desde un $20 \%$ hasta un $71,2 \%$ de los pacientes con $\mathrm{ES}^{9,10}$. La disminución de la secreción salival debido a la fibrosis de las glándulas salivales genera disfagia orofaríngea al disminuir la lubricación del bolo alimenticio y el tránsito del mismo por la faringe y el esófago?.
Las opciones terapéuticas suelen estar limitadas a modificaciones dietarias con porciones pequeñas de alimentos, alimentos blandos y el aumento de la ingesta de líquidos. Se recomienda un control odontológico periódico y una óptima higiene bucal. Al día de la fecha, no existen datos de que las manifestaciones orofaríngeas en los pacientes con ES respondan a un tratamiento médico específico 9 .

Está comúnmente aceptado que un subgrupo de pacientes con ES presentan además manifestaciones clínicas de otras enfermedades del tejido conectivo como Miopatías Inflamatorias, Lupus Eritematoso Sistémico, Artritis Reumatoidea o Síndrome de Sjögren. Estos pacientes han sido habitualmente clasificados como "síndromes de superposición”. Este concepto es un concepto complicado ya que no existen criterios uniformes que permitan clasificar al paciente como tal. Comúnmente se dice que si un paciente con ES definida cumple al mismo tiempo con los criterios de clasificación de otra de estas enfermedades autoinmunes, tiene un síndrome de superposición?

En el caso de los síntomas sicca de la ES, la fisiopatogenia es diferente a los síntomas sicca del Síndrome de Sjögren (SS). En la ES, como se menciona más arriba, la fibrosis de las glándulas salivales es lo que produce la disminución de la secreción salival, mientras en el SS, la infiltración crónica linfocitaria es la causa de la disfunción glandular ${ }^{11}$.

En un estudio francés, publicado en el año 2006 por Avouac y cols., se consideró que un paciente presentaba SS asociado a ES sólo si en la biopsia glandular se encontraba sialoadenitis linfocítica con uno o más foco score (grado 3 o 4 de la escala de Chisholm y Mason). El solo hecho de cumplir criterios clasificatorios de SS en un paciente con ES no sería considerado suficiente, dado que la presencia de síntomas sicca en ES es muy frecuente, como se mencionó previamente, y la presencia de anti-Ro y anti- 
La no sería suficiente para clasificar al paciente como SS asociado $^{11}$. En este estudio, un $20 \%$ de las biopsias de glándulas salivales de pacientes con ES y síntomas sicca presentaron histología con un grado 3 o 4 de Chisholm y Mason (SS asociado). Y en dos de estos pacientes se observó en la biopsia la presencia simultánea de fibrosis y de sialoadenitis linfocitaria (hallazgos de ES y Sjögren en la misma glándula $)^{11}$. Se observó que la forma limitada de ES, la presencia de anticuerpos anticentrómero y de factor reumatoideo se asociaron a la presencia de SS asociado a $E^{11}$. Por otro lado, es reconocida la asociación entre la forma limitada de ES con SS y Cirrosis Biliar Primaria ${ }^{12}$.

En este número de la Revista Argentina de Reumatología se presenta un trabajo brasileño realizado por Coelho Harimoto y cols., en donde se analiza la presencia de síndrome de Sjögren asociado en una cohorte de 69 pacientes con Esclerosis Sistémica. De estos pacientes, 16 $(23,2 \%)$ cumplían criterios de clasificación de SS del año $2002^{13}$ y un $62,3 \%$ presentaron xerostomía.

Si bien en el estudio no se encontraron diferencias clínicas entre aquellos pacientes con ES y SS asociado comparados con aquellos con ES sola, este artículo resulta de interés para conocer la realidad epidemiológica de esta entidad en un país con distintas características poblacionales que la Argentina.

\section{Bibliografía}

1. LeRoy EC, Black C, Fleischmajer R, et al. Scleroderma (systemic sclerosis): classification, subsets and pathogenesis. J Rheumatol. 1988;15(2):202-205.

2. Clements PJ, Becvar R, Drosos AA, Ghattas L, Gabrielli A. Assessment of gastrointestinal involvement. Clin Exp Rheumatol. 2003;21(3 Suppl 29):S15-8.

3. Forbes A, Marie I. Gastrointestinal complications: the most frequent internal complications of systemic sclerosis. Rheumatology. 2009;48(Supplement 3):iii36iii39.

4. Steen VD, Medsger TA. Changes in causes of death in systemic sclerosis, 1972-2002. Ann Rheum Dis. 2007;66(7):940-944.

5. Scolnik M, Lancioni E, Saucedo C, et al. Systemic sclerosis in Argentina: evaluation of a large cohort from a single centre and comparison with other international series. Clin Exp Rheumatol. 32(6 Suppl 86):S-94-7.

6. Vitali C, Borghi E, Napoletano A, et al. Oropharyngolaryngeal Disorders in Scleroderma: Development and Validation of the SLS Scale. Dysphagia. 2010;25(2):127-138.

7. Scardina GA, Mazzullo M, Messina P. [Early diagnosis of progressive systemic sclerosis: the role of oro-facial phenomena]. Minerva Stomatol. 51(7-8):311-317.
8. Domsic R, Fasanella K, Bielefeldt K. Gastrointestinal Manifestations of Systemic Sclerosis. Dig Dis Sci. 2008;53(5):1163-1174.

9. Clarke, John O; Hirano I. Scleroderma: From Pathogenesis to Comprehensive Management. First Edit. (Varga, John; Denton, Christopher P; Wigley FM, ed.). Springer; 2012.

10. Ramos-Casals M, Brito-Zerón P, Font J. The Overlap of Sjögren's Syndrome with Other Systemic Autoimmune Diseases. Semin Arthritis Rheum. 2007;36(4):246-255.

11. Avouac J, Sordet C, Depinay C, et al. Systemic sclerosisassociated Sjögren's syndrome and relationship to the limited cutaneous subtype: Results of a prospective study of sicca syndrome in 133 consecutive patients. Arthritis Rheum. 2006;54(7):2243-2249.

12. Nakamura T, Higashi S, Tomoda K, Tsukano M, Sugi K. Primary biliary cirrhosis (PBC)-CREST overlap syndrome with coexistence of Sjögren's syndrome and thyroid dysfunction. Clin Rheumatol. 2007;26(4):596600.

13. Vitali C, Bombardieri S, Jonsson R, et al. Classification criteria for Sjögren's syndrome: a revised version of the European criteria proposed by the American-European Consensus Group. Ann Rheum Dis. 2002;61(6):554558. 